Published in final edited form as:

Nucl Instrum Methods Phys Res A. 2011 August 21; 648 Supplement 1: S281-S283. doi:10.1016/j.nima. 2010.11.053.

\title{
Carbon Nanotube based X-ray Sources: Applications in Pre- Clinical and Medical Imaging
}

\author{
Yueh Z. Lee, MD/PhD ${ }^{1,2}$, Laurel Burk ${ }^{2}$, Ko-han Wang, B.S. ${ }^{1}$, Guohua Cao, PhD $^{2}$, Jianping \\ Lu, $\mathrm{PhD}^{3}$, and Otto Zhou, $\mathbf{P h D}^{2,3}$ \\ ${ }^{1}$ Department of Radiology, University of North Carolina at Chapel Hill, Chapel Hill, NC 27599, \\ USA \\ 2Department of Physics and Astronomy, University of North Carolina at Chapel Hill, Chapel Hill, \\ NC 27599, USA \\ ${ }^{3}$ Curriculum in Applied Science and Engineering, University of North Carolina at Chapel Hill, \\ Chapel Hill, NC 27599, USA
}

\section{Introduction}

Carbon nanotube (CNT) based x-ray sources generate electrons through field emission with a cathode of vertically arranged nanotubes. The high aspect-ratio and near atomic sharpness of CNT tips allows them to serve as near optimal field-emission cathodes. The application of an electric field across the nanotube creates electron emission at $5 \mathrm{~V} / \mu \mathrm{m}$ or lower, with stable current densities of $1500 \mathrm{~mA} / \mathrm{cm}^{-2}$ or higher. [1] Source stability at high currents has been demonstrated to be in excess of 30 continuous hours of beam on time, depending on operating conditions and with focal spots as small as 50 microns. Even smaller focal spots are possible, with the tradeoff of maximum current.

Traditional thermionic $\mathrm{x}$-ray sources require additional gate suppression or mechanical shutters to create millisecond scale $\mathrm{x}$-ray pulses. In contrast, field emission cathodes allow for simple modulation of the $\mathrm{x}$-ray through gate voltage modulation. For pre-clinical small animal imaging applications, this allows straightforward respiratory or cardiac gated imaging at high physiological rates. For example, mice usually respire at over 100 breaths per minute and have cardiac cycles on the order of 300 beats per minute or higher.

\section{Preclinical Imaging}

We have developed a number of prototype micro-CT systems using the CNT x-ray sources. Our most current system consists of a custom compact CNT x-ray source operated at $50 \mathrm{kVp}$ and $2 \mathrm{~mA}$ anode current. [2,3] The tube has a tungsten anode, internal electron focusing and a $100 \mu \mathrm{m}$ effective focal spot size. The CsI based flat panel detector (C7940DK-02, Hamamatsu Photonics, Japan) was mounted opposite the x-ray source on a Huber 430 goniometer (Huber Diffraktiontechnik, Germany) to allow for rotation of the source-detector pair around the animal. A custom animal bed and x-ray shielded enclosure were also made

\footnotetext{
(C) 2010 Elsevier B.V. All rights reserved.

Corresponding Author: Yueh Z. Lee, The University of North Carolina at Chapel Hill, Phillips Hall, CB \#3255, Chapel Hill, NC 27599-3255, yueh@alum.mit.edu, Fax: 919-962-0480, Phone: 919-966-1461.

Publisher's Disclaimer: This is a PDF file of an unedited manuscript that has been accepted for publication. As a service to our customers we are providing this early version of the manuscript. The manuscript will undergo copyediting, typesetting, and review of the resulting proof before it is published in its final citable form. Please note that during the production process errors may be discovered which could affect the content, and all legal disclaimers that apply to the journal pertain.
} 
to facilitate anesthesia delivery and streamline animal setup. The most recent system is seen in Figure 1, nicknamed "Charybdis".

We have demonstrated cardiac and respiratory gated imaging at 12 and $30 \mathrm{msec} \mathrm{x}$-ray pulses, respectively, allowing functional imaging of mouse models of pulmonary and cardiac disease, Figures $2-5$. Images were acquired using gaseous anesthesia, without the need of intubation or complex animal setup. The respiratory trace was derived from a bellows placed underneath the abdomen of the animal. The electrocardiogram signal was derived from three neonatal leads attached to the animal. Iodinated blood-pool contrast agent (Fenestra VC, ART Advanced Research Technologies, Canada) was administered intravenously for the cardiac models to help better delineate the blood volume.

Figure 2 demonstrates an axial image of a normal, wild-type mouse lung, acquired during inspiration, at the peak of the respiration pressure curve. Figure 3 demonstrates an LUL+/ Kras+ multi-focal lung tumor model, where multiple tumors may be seen as rounded masses. Traditionally, these tumor models are followed by optical imaging to look at the activation of an optical reporter gene. In contrast, human tumors are followed clinically using traditional computed tomography. Our early work with this model quickly demonstrates that remodeling of the lung volumes were occurring, a detail that is almost impossible to derive from traditional optical or even histological techniques.

Figure 4 demonstrates axial images of the mouse heart after the injection of the intravenous contrast agent. The images in Figure 4 were acquired at $0 \mathrm{msec}$ and $75 \mathrm{msec}$ after the electrical R-wave of the heart, and correspond to the mechanical diastole and systole phases of the cardiac cycle, respectively. Figure 5 demonstrates preliminary work on delayed enhancement cardiac micro-CT for evaluation of myocardial infarction. The rim of hyperenhancement along the myocardium of the left ventricle represents an area of infarcted tissue, in a mouse model with coronary artery ligation and reperfusion. Clinically, this implies that the heart tissue is not revivable, even if blood flow is restored to this region.

Minimum imaging time of the current system is 8 minutes, primarily limited by detector read-out times combined with gantry rotation. Most respiratory imaging studies require approximately 9 to 10 minutes of imaging time, while cardiac imaging requires 12 to 15 minutes, due to the need for synchronization of both the cardiac and respiratory signals at the appropriate phase. Physiological parameters, such as tidal lung volume and ejection fraction, can be readily derived from the CNT $\mu \mathrm{CT}$ images. No intubation of the animals is required, reducing setup time and trauma. In contrast to commercially available systems, the CNT micro CT system enables rapid prospectively gated imaging with a straightforward control system.

\section{Clinical Applications}

Clinical applications of the CNT x-ray sources are primarily enabled by the ability to place multiple $\mathrm{x}$-ray sources in close proximity to allow for the creation of multiple individually addressable x-ray sources. Tomosynthesis allows more depth resolution and improves separation between overlying objects. We have demonstrated this application for stationary digital breast tomosynthesis, with the creation of a linear array of x-ray sources. [4] A traditional single source based breast tomosynthesis system requires moving the source to multiple locations, typically 11 to 25 . This results in blurring of the focal spot due to the required motion for multiple projections. Using multiple stationary sources instead, however, minimizes focal spot motion, reducing the apparent focal spot and improving resolution. Additional information about this clinical application appears in a separate article in this same issue. 
The addressable arrays may also be utilized for other novel volumetric imaging in complex geometries, such as the ongoing development for radiotherapy and interventional applications. Image guided radiotherapy can use a separate $\mathrm{x}$-ray source to provide anatomic localization during treatment, typically using two projections. [5] In contrast, using a twodimensional array of sources allows the creation of tomographic images, with increased spatial information as compared to the projection images.

\section{Conclusion}

CNT based X-ray sources have allowed the development of new imaging techniques and geometries not previously attainable using conventional sources. In this brief overview, we demonstrate preclinical applications of these sources in pulmonary and cardiac mouse models of human diseases that allow rapid, gated imaging. Furthermore, new clinical applications are already being developed which take advantage of the ability to close-pack these x-ray sources. We feel that many other applications may also be developed, breaking beyond the bounds of the traditional thermionic sources.

\section{Acknowledgments}

The mouse lung tumor model was provided by Dr. William Y. Kim of the Lineberger Cancer Institute at The University of North Carolina at Chapel Hill. Cardiac mouse models were provided by Drs. Eunice Kang, Monte Willis and Mauricio Rojas of the McCallister Heart Institute at The University of North Carolina at Chapel Hill. The authors acknowledge support from NIH-NCI (U54CA119343), NIH-NIBIB (4R33EB004204-01), UNC University Cancer Research Fund, and Xintek, Inc.

\section{References}

1. Calderón-Colón X, Geng H, Gao B, An L, Cao G, Zhou O. Nanotechnology. 2009; 20325707.

2. Liu Z, Yang G, Lee YZ, Bordelon D, Lu J, Zhou O. Applied Physics Letters. 2006; 89:103111103113.

3. Cao G, Lee YZ, Peng R, Liu Z, Rajaram R, Calderón-Colón X, An L, Wang P, Phan T, Sultana S, Lalush DS, Liu JP, Zhou O. Physics in Medicine and Biology. 2009; 54:2323-2340. [PubMed: 19321922]

4. Qian X, Rajaram R, Calderón-Colón X, Yang G, Phan T, Lalush DS, Liu JP, Zhou O. Medical Physics. 2009; 36:4389-4399. [PubMed: 19928069]

5. Maltz JS, Sprenger F, Fuerst J, Paidi A, Fadler F, Bani-Hashemi AR. Medical Physics. 2009; 36:1624-1636. [PubMed: 19544779] 


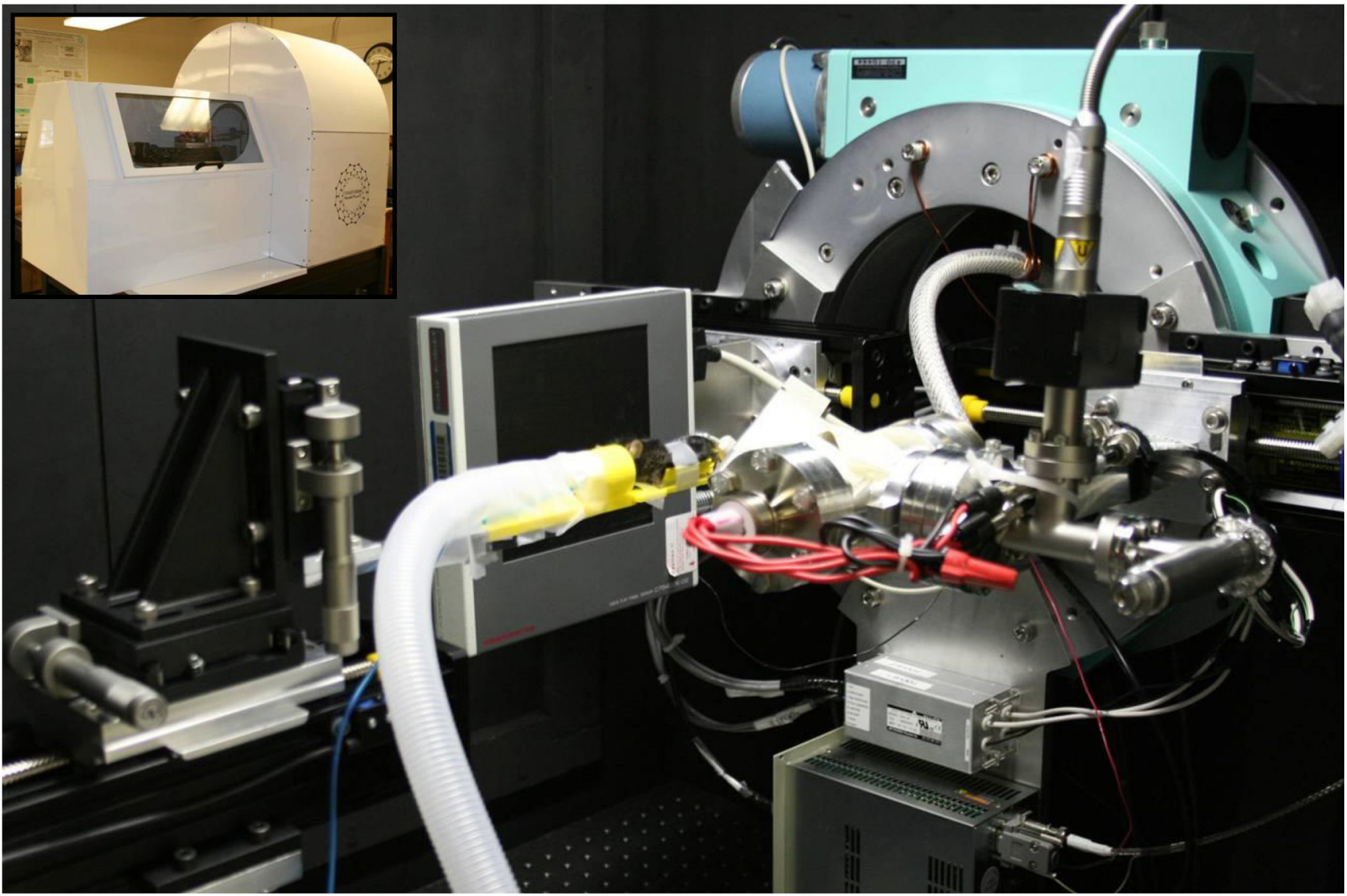

Figure 1.

Custom CNT micro-CT based micro-CT, nicknamed "Charybdis". The inset photograph in the top left shows the device with its custom cover. 


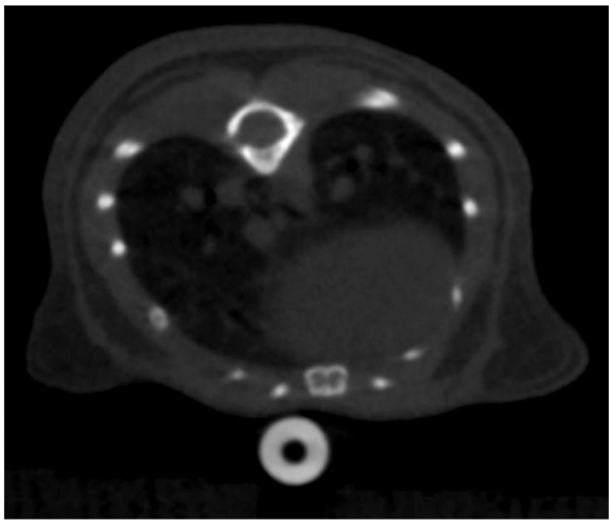

Figure 2.

Axial CNT respiratory gated micro-CT image of the lower thorax of a wild-type mouse without contrast demonstrating visualization of the lung bases. 


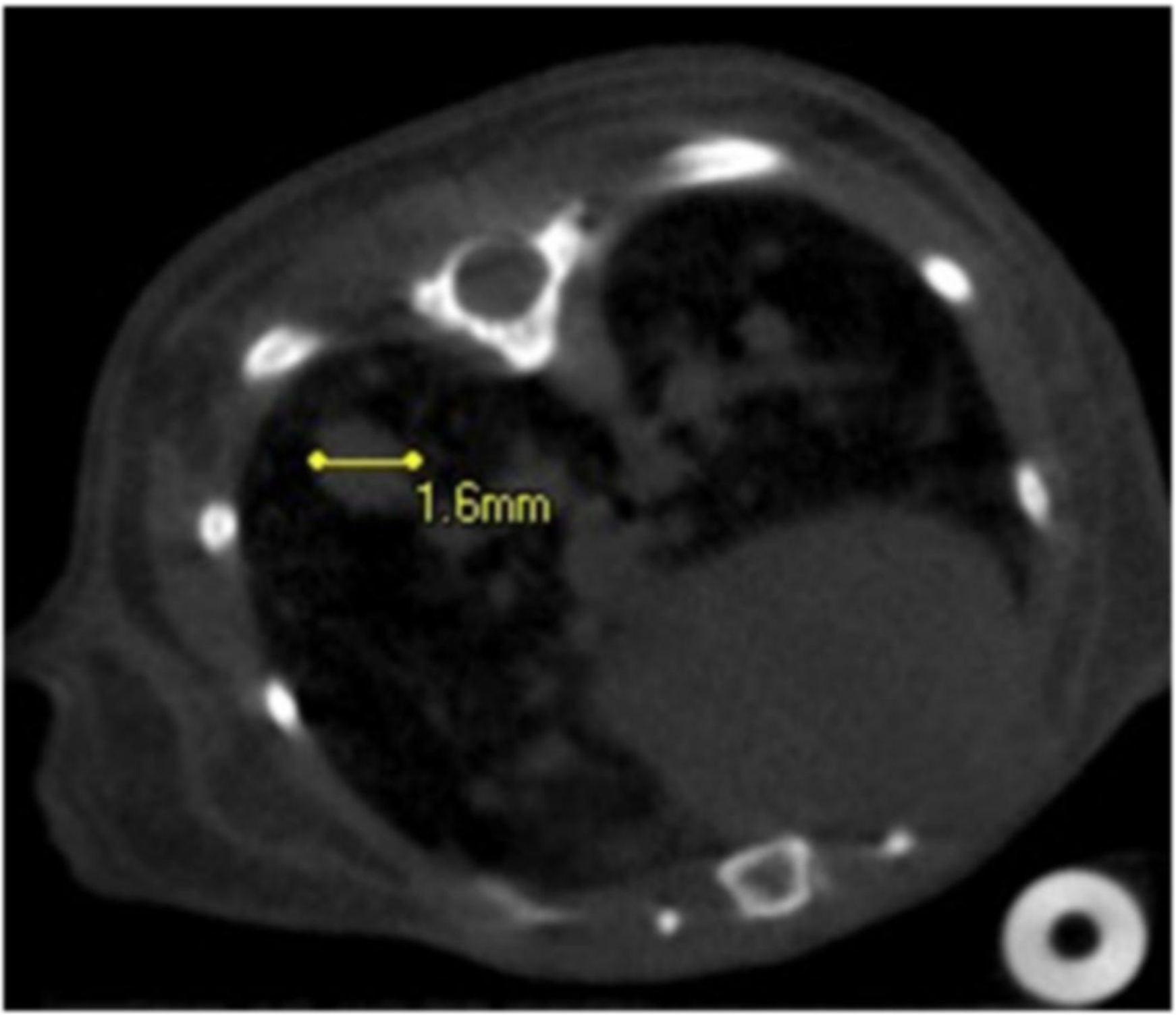

Figure 3.

Axial CNT respiratory gated micro-CT image of the lower thorax of mouse model of multifocal lung tumors. The largest lesion at the right lung base (left of the image) is identified and measures $1.6 \mathrm{~mm}$. Numerous additional tumors are also noted. 


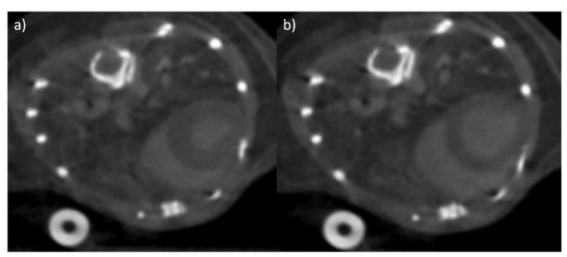

Figure 4.

Axial CNT cardiac gated micro-CT images of the heart of a wild-type mouse after the administration of intravenous contrast at two different time points during the cardiac cycle, representing mechanical diastole (a) and systole (b). 


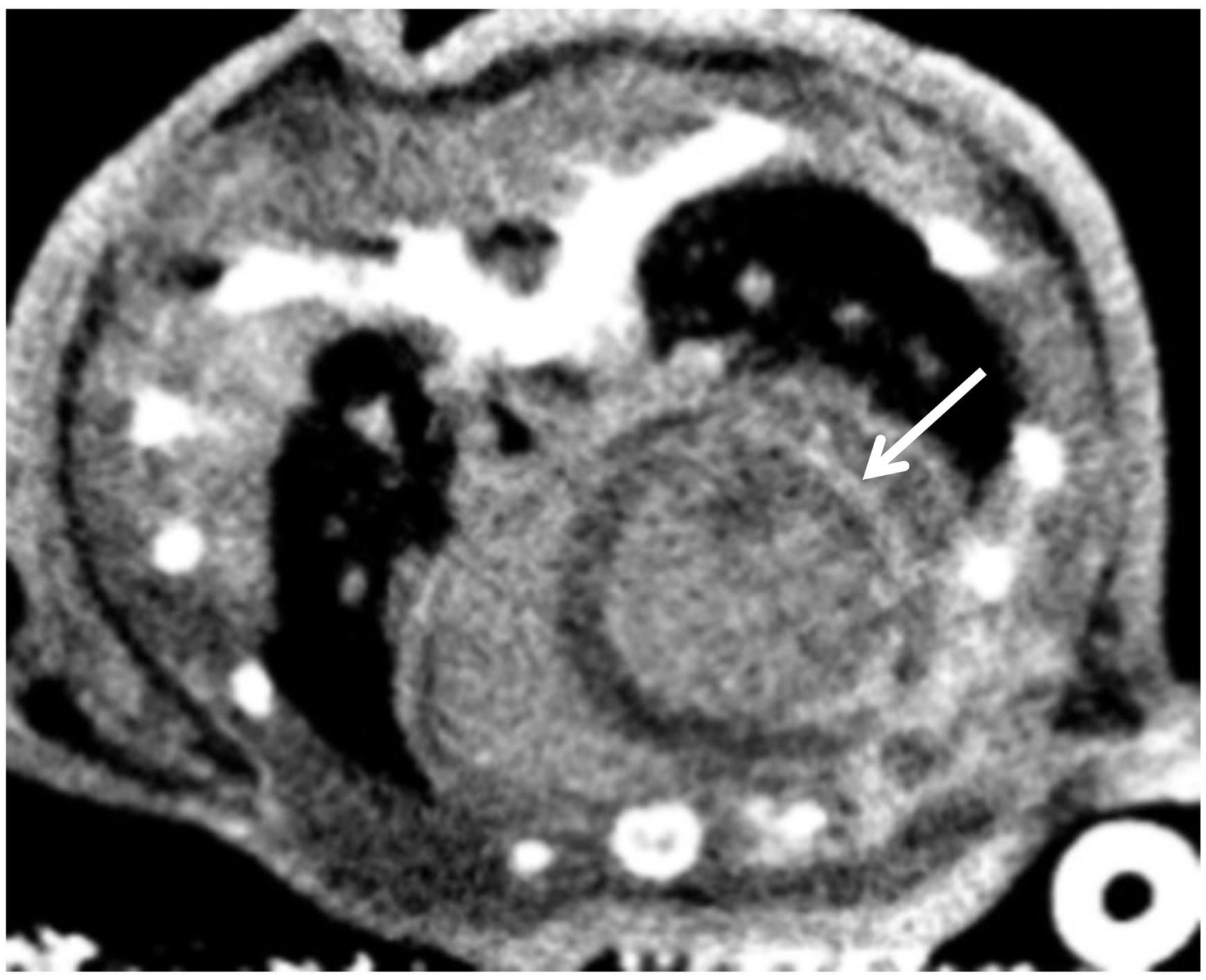

Figure 5.

Axial CNT cardiac micro-CT image of the heart of a cardiac ischemia/reperfusion model demonstrating hyperenhancement (arrow) of the lateral left ventricle wall, representing an area of myocardial infarction. 